\title{
UNITARY SYSTEMS AND BESSEL GENERATOR MULTIPLIERS
}

\author{
DEGUANG HAN AND DAVID R. LARSON
}

\begin{abstract}
A Bessel generator multiplier for a unitary system is a bounded linear operator that sends Bessel generator vectors to Bessel generator vectors. We characterize some special (but useful) Bessel generator multipliers for the unitary systems that are ordered products of two unitary groups. These include the wavelet and the Gabor unitary systems. We also provide a detailed exposition of some of the history leading up to this work.
\end{abstract}

\section{INTRODUCTION}

Orthonormal wavelets can be regarded as complete wandering vectors for a special system of unitary operators acting on a separable infinite dimensional Hilbert space. In the standard wavelet (and frame-wavelet) theory the system is an ordered product $\left\{D^{n} T^{k}: n \in \mathbb{Z}, k \in\right.$ $\left.\mathbb{Z}^{d}\right\}$ of two unitary groups $\left\{D^{n}\right\}$ and $\left\{T^{k}\right\}$ acting on the Hilbert space $L^{2}\left(\mathbb{R}^{d}\right)$ called the dilation group and the translation group, respectively, which intertwine in their action on $H$ in a particular way. The standard Gabor frame theory has a similar operator model, with the difference being in the way the groups intertwine. In the standard wavelet theory both groups are abelian, with the dilation group being cyclic with generator $D$ corresponding to dilation of functions by a $d \times d$ invertible real matrix on $L^{2}\left(\mathbb{R}^{d}\right)$, and with the translation group having $d$ generators corresponding to translation of functions by the elements of a basis of $\mathbb{R}^{d}$. In the standard Gabor theory the groups are the modulation group and the translation group. In the Gabor theory the groups commute modulo scalars, but in the wavelet theory they do not. There have been several generalizations of these theories by introducing other natural candidates for the dilation, translation, and modulation groups, and considering systems which are ordered products of more than two groups.

If we let $\mathcal{U}_{D, T}$ denote the wavelet unitary system with $D$ the dilation unitary group and $T$ the translation unitary group, then an orthonormal wavelet (resp. Riesz wavelet) can be thought as an orthonormal (resp. Riesz) basis generator vector for $\mathcal{U}_{D, T}$ and a frame wavelet can be viewed as a frame generator vector for $\mathcal{U}_{D, T}$. These have been studied in many previous papers in the literature. We will call a vector $x$ a Bessel wavelet if the action of $\mathcal{U}_{D, T}$ on $x$ generates a Bessel sequence; i.e. if $x$ is a Bessel sequence generator for $\mathcal{U}_{D, T}$. It turns out that one can gain much additional perspective on wavelets and frame wavelets if one views them as special cases of Bessel wavelets. One reason for this is that the set of Bessel wavelets is a linear space, unlike the sets of wavelets and frame wavelets. When one puts a certain natural norm on the space of Bessel wavelets, the Bessel bound norm, in addition to the usual norm as a vector in Hilbert space, it becomes isomorphic to

\footnotetext{
2000 Mathematics Subject Classification. Primary 42C15, 46C05, 47B10.

Key words and phrases. unitary system, Bessel generator multiplier, frame, Parseval dual, frame representation, Gabor frame, lattice tiling .
} 
an operator space in the usual sense of the term. Thus it admits some investigation using techniques of modern operator algebra. Some new results for wavelets and frame wavelets, and some new proofs of previously known results, can then be obtained because of their role as very special elements of this Bessel wavelet operator space. There is nothing sacred about wavelet theory in this approach. Gabor Bessel generators are defined in the same way for the Gabor unitary systems, and they too are operator spaces in a natural way. More general unitary systems (in its greatest generality a unitary system is simply defined as a discrete collection of unitary operators that contains the identity operator $I$ ) can also be associated with operator spaces in this way.

A Bessel generator multiplier for a unitary system is a bounded linear operator that sends Bessel generator vectors to Bessel generator vectors. In this article we will characterize some special (but useful) Bessel generator multipliers for the unitary systems that are ordered products of two unitary groups. These include the wavelet and the Gabor unitary systems discussed above. These results are new and have not been published elsewhere. We first defined Bessel generator multipliers in the earlier paper [22], where we used them to give a new proof of a theorem of Bownik [2] showing that the set of wavelet frames for a fixed wavelet unitary system $\mathcal{U}_{D, T}$ are, indeed, dense in the underlying Hilbert space $L^{2}\left(\mathbb{R}^{d}\right)$. Here $D$ is the dilation group corresponding to dilation by an expansive matrix. This answered a question of the second author (c.f. [31, 32]). More precisely, we first worked out the new proof of Bownik's result and then we realized that it could be made more crystal clear by introducing the concept of a Bessel generator multiplier. In this article we first give an exposition of our work in [22], and then we prove our new results.

We recall and introduce some notations and definitions that will be needed in this paper.

A frame for a Hilbert space $H$ is a sequence of vectors $\left\{f_{j}\right\}_{j \in \mathbb{J}}$ in $H$ such that there exist positive constants $A$ and $B$ such that

$$
A\|f\|^{2} \leq \sum_{j \in \mathbb{J}}\left|\left\langle f, f_{j}\right\rangle\right|^{2} \leq B\|f\|^{2}
$$

holds for every $f \in H$, where $\mathbb{J}$ is a countable index set. We call $A$ a lower frame bound and $B$ an upper frame bound. The optimal lower and upper bounds are called the frame bounds. A tight frame refers to a frame when both upper and lower frame bounds are the same, and a Parseval frame is a frame whose frame bounds are one. If we only require the right hand inequality in the frame definition, then $\left\{f_{n}\right\}$ is called a Bessel sequence and the optimal $B$ is called the Bessel bound. The analysis operator $\Theta$ for a Bessel sequence is defined by

$$
\Theta(f)=\sum_{j \in \mathbb{J}}\left\langle f, f_{j}\right\rangle e_{j}, \quad f \in H
$$

where $\left\{e_{j}\right\}$ is the standard orthonormal basis for the $\ell^{2}(\mathbb{J})$-sequence space. It is easy to verify that $\Theta^{*} \chi_{j}=f_{j}, j \in \mathbb{J}$, and that $\Theta^{*} \Theta=\sum_{j} f_{j} \otimes f_{j}$, where $f \otimes g$ is the elementary tensor rank-one operator defined by $(f \otimes g)(h)=\langle h, g\rangle f$. Moreover, a sequence $\left\{f_{j}\right\}$ is a frame (resp. a Bessel sequence) if and only if $\sum_{j \in \mathbb{J}} f_{j} \otimes f_{j}$ is weakly convergent to a bounded invertible operator (resp. bounded operator) on $H$. Two Bessel sequences $\left\{f_{j}\right\}$ and $\left\{g_{g}\right\}$ are called strongly disjoint or orthogonal if the range spaces of their analysis operators are 
orthogonal, i.e.,

$$
\sum_{j \in \mathbb{J}}\left\langle f, f_{j}\right\rangle\left\langle g_{j}, g\right\rangle=0, \quad \forall f, g \in H .
$$

There is an interesting characterization of orthogonal Parseval frames in terms of the frame property for orthogonal direct sum spaces:

Proposition 1.1. [20] Let $\left\{f_{j}\right\}$ and $\left\{g_{g}\right\}$ be two Parseval frames for $H$ and $K$, respectively. Then $\left\{f_{j}\right\}$ and $\left\{g_{g}\right\}$ are strongly disjoint if and only if $\left\{f_{j} \oplus g_{j}\right\}$ is a Parseval frame for the orthgonal direct sum space $H \oplus K$.

The investigation of frames with special structures has been the main focus in the literature of frame theory. Included are the wavelet frames, the Gabor (or Weyl-Heisenberg) frames, and the frames obtained by the action of discrete group representations. All of these frames can be obtained by applying a collection of unitary operators, called a unitary system, to a generating vector in the representing Hilbert space. Such a vector $x \in H$ is called a (complete) wandering vector (resp a frame generator vector, tight frame generator or a Bessel generator vector for a unitary system $\mathcal{U}$ if $\mathcal{U} x:=\{U x\}_{U \in \mathcal{U}}$ (as a sequence indexed by $\mathcal{U}$ ) is an orthonormal basis for $H$ (resp. a frame, tight frame or a Bessel sequence). If a bounded linear operator $A$ on $H$ maps every wandering vector of $\mathcal{U}$ to a wandering vector of $U$, then it is called a wandering vector multiplier. Wandering vector multipliers play a significant role in studying the topological properties of of the set of all wavelets. For example, a complete characterization of the wavelet multipliers $A$ of the form $\hat{A}=M_{h}$ was obtained in [39] and was used to show that the set of all MRA wavelets is path-connected. Here $\hat{A}$ denotes the Fourier transform of $A$ and $M_{h}$ denotes the multiplication unitary operator associated with the unimodular function symbol $h$. It was shown in $[21,27]$ that when the unitary system is a group, then the set of all wandering vector multipliers form a group. A complete characterization of the wandering vector multipliers for abelian and for ICC groups is also given in [21]. However, in general it is difficult to characterize the wandering vector multipliers even when the unitary system $\mathcal{U}$ has a relatively simple structure.

If a bounded linear operator $A$ on $H$ maps every frame generator vector for $\mathcal{U}$ to a frame generator vector of $U$, then it is called a frame generator multiplier. Similarly, if $A$ maps the set of Bessel generator vectors for $U$ into itself it is called a Bessel generator multiplier.

As mentioned above, the set of wandering vector multipliers can have a complex structure even when the unitary system is relatively simple. The set of frame generator multipliers likewise has a complex structure. The wandering vector multipliers form a unitary semigroup, and in some special cases this is a group. But proofs can be difficult $[21,27]$. The frame generator multipliers naturally form a multiplicative semigroup, but even in relatively simple cases not much is known about the structure.

On the other hand, it is easily seen that the set of Bessel generator vectors for a unitary system $\mathcal{U}$, denoted by $\mathcal{B}_{\mathcal{U}}$, is closed under the taking of linear combinations so is a linear space. It follows that the set of Bessel generator multipliers for $\mathcal{U}$ is an algebra of operators in $B(H)$. It is not generally norm closed, and it will not generally be self-adjoint. We will show that the Bessel bound is a complete norm on the space of Bessel generator vectors, making it a Banach space. In fact it is an operator space, which can be seen in more than one natural way. The operator space structure resulting from these different natural representations 
are equivalent even though the constructions in the representations are different. We will show that a Bessel generator multiplier $A$ (which is a bounded linear operator on $H$ ) is also bounded as a linear map from $\left(\mathcal{B}_{\mathcal{U}}\right.$, the bessel bound norm) into itself. Thus the algebra of Bessel generator multipliers carries two norms: the usual operator norm from $\mathrm{B}(\mathrm{H})$, and the operator norm from the Banach space $\left(\mathcal{B}_{\mathcal{U}}\right.$, the Bessel bound norm) into itself. These norms are frequently different.

A Bessel generator multiplier $A$ does not usually commute with the action of the unitary system, so while it induces a bounded endormorphism of the operator space $\mathcal{B}_{\mathcal{U}}$, it is not usually a simple endormorphism of the type induced by a multiplication operator on a Banach algebra. If $x$ is in $\mathcal{B}_{\mathcal{U}}$ and $y=A x$ then the Bessel sequence $\mathcal{U} y$ generated by the action of the unitary system $\mathcal{U}$ on $y$ is generally not the sequence of vectors indexed by $\mathcal{U}$ obtained by multiplying the sequence $\mathcal{U} x$ on the left by $A$. The action of $A$ on $y$ is in a sense the action of $A$ on $x$ twisted by the action of the unitary group on $A$. This twisting is what makes it interesting, and makes the structure of the generator multiplier algebra rather rich. There will be no twisting if and only if $A$ actually commutes with the unitary system $\mathcal{U}$. We will give some examples to illustrate this. There are some interesting open questions involving the operator space structures of the space of Bessel generator vectors and algebra of Bessel generator multipliers. We will present some results in this direction, and pose some open questions in this direction.

This article was written for inclusion in a special volume of proceedings of the conference Twenty Years of Wavelets, Chicago, May 2009. The second author thanks the organizers for inviting him to be a plenary speaker.

\section{Density and Connectivity of Wavelet Frames}

As we mentioned in the introduction, one of the motivations for studying Bessel vector multipliers comes from its connection with the density and connectivity problems for wavelet frames. In a recent paper [22] we showed that the algebra of all Bessel generator multipliers for a wavelet system carries a rich algebraic and topological structure that can be used to give a solution to the path-connectivity problem and the density problem for wavelet frames. Bownik solved these problems in [2]. We gave an alternate solution which is more transparent in some ways. Since the wavelet Bessel generator multipliers were proven useful, and with seemingly math-e-magical properties, in the study of certain properties of wavelets, we were led to consider a more complete investigation of their properties, not only for wavelet systems but for other natural unitary systems.

The question of whether the set of all Riesz wavelets for the dyadic wavelet system on $L^{2}(\mathbb{R})$ is a norm-dense path-wise connected subset of $L^{2}(\mathbb{R})$ was asked in [29] by the second author. This question is related to the problem that was posed by Guido Weiss and his group $[25,26]$ and independently by Dai and Larson ([8], problem 1) of whether the set of all orthonormal dyadic wavelets is connected. Neither conjecture has been settled to date, although it has been shown [39] that the set of $M R A$ orthonormal dyadic wavelets is connected. Shortly after this, the two authors of this paper developed an operator-theoretic approach to frame theory in [20], and the same problems (density and connectivity) were posed for wavelet frames (also called frame wavelets or framelets). While this density problem was not posed formally in [20] it was alluded to in that memoir, and also in the 
semi-expository papers [30] and [32], and it was finally posed formally (along with some other related problems) in [31]. However, it was pointed out in [20] that for the Gaborframe case, and for the group-frame case (i.e. frame sequences generated by the action of a unitary group on a single generator vector), both the density and the connectivity problems have positive solutions. This follows from the parametrization theorem for frame vectors in terms of the set of all invertible operators from the von Neumann algebra generated by the unitary system. It was later proven that the parametrization result presented in [20] is also valid and very useful for the more general projective unitary group representations (cf. $[10,12,13,18]$ ). These systems were also called group-like systems, and they include the Gabor systems as special cases. The frame density and connectivity problems also have positive solutions for these systems. The general connectivity and density problems for the set of wavelet frames remained open until recently Bownik settled both problems affirmatively.

Theorem 2.1. [2] The set all wavelet frames is dense and path-connected in $L^{2}\left(\mathbb{R}^{d}\right)$.

Bownik in fact proved the density of wavelet frames not only in the Hilbert space norm but also in another natural logmodular norm associated with wavelet theory (see [36] and [17] for the definition of this norm). While the general connectivity and density problems are settled for the case of wavelet frames, they are still open for the case of wavelets. We point out that before Bownik's path connectivity result, there has been steady progress on the connectivity problem for special classes of wavelets and wavelet frames, including the MSF (s-elementary) and MRA (multiresolution analysis) wavelets and their analogues for wavelet frames and especially Parseval wavelets. The interested reader should refer to (c.f. $[4,6,7,14,15,16,23,33,34,37,39])$ for details and exposition of these results. Bowink's result does not overlap with the results for special classes of wavelet frames since it is usually more difficult and challenging to construct or prove the existence of continuous paths in a subset of wavelet frames.

In [22] we re-examined the density and connectedness results of Bownik and observed that the essential ingredient that makes the proofs work is the frame-orthogonality (or strongdisjointness), a concept that arose simultaneously and independently to Balan [1] and the authors some time ago [20]. This is a natural geometric concept in frame theory which was formally introduced and studied in $[1,20]$, and it has proven useful for developing the theory of frames and its applications. While Bownik does not explicitly use term frameorthogonality in his argument, it is clear that the essential reason the argument works seems to involve the orthogonality concept. This was made crystal-clear in the argument of [22] where a key observation is that if a wavelet Bessel function $f$ is strongly disjoint with a wavelet frame $\psi$, then $f+t \psi$ is a wavelet frame for all $t \neq 0$ and hence $f$ is the limit of a sequence of wavelet frames. This allows us to obtain a new type of approximation result for wavelet Bessel sequence generators.

Lemma 2.2. [22] Assume that $\psi$ is wavelet frame and $g$ is Bessel. If $\psi$ and $g$ are strongly disjoint, then $\varphi=\psi+g$ is also a wavelet frame. Moreover, if $\psi$ has upper and lower frame bounds $A$ and $B$, respectively, and $g$ has a Bessel bound $C$, then $\varphi$ has a lower frame bound $A$, and upper frame bound $B+C$. 
With the help of Lemma 2.2, we obtained the following stronger version of Bownik's density theorem.

Theorem 2.3. [22] (i) Every function $f \in L^{2}(\mathbb{R})$ is a limit of as asymptotically tight sequence of wavelet frames, i.e., . there exists a sequence of wavelet frames $\left\{\psi_{n}\right\}$ such that $\lim _{n \rightarrow \infty} \frac{B_{n}}{A_{n}}=1$ and $\lim _{n \rightarrow \infty} \psi_{n}=f$, where $B_{n}$ and $A_{n}$ are the upper and lower frame bounds of $\psi_{n}$.

(ii) Assume that $\psi$ is a wavelet frame and $f \in L^{2}(\mathbb{R}) \cap L^{\infty}(\mathbb{R})$. Then there exists a continuous path $\psi_{t}$ such that $\psi_{0}=\psi, \psi_{1}=f$ and $\psi_{t}$ is a wavelet frame for every $0 \leq t<1$. In particular, this also implies that the set of all wavelet frames is path-connected and is dense in $L^{2}(\mathbb{R})$.

All these results still hold for wavelet frames with expansive dilation matrices and integer lattice $\mathbb{Z}^{d}$-translations in $L^{2}\left(\mathbb{R}^{d}\right)$.

Theorem 2.3 (i) seems indicate that the set of all tight wavelet frames might be dense in $L^{2}(\mathbb{R})$. This was proven to be false by Bownik in [3]. In fact he proved that if a function $\psi \in L^{2}(\mathbb{R})$ is in the closure of the set of all tight frames, then it satisfies one of the familiar equations characterizing Parseval wavelet frames, i.e.,

$$
\sum_{j=o}^{\infty} \hat{\psi}\left(2^{j}\right) \overline{\hat{\psi}\left(2^{j}(\xi+q)\right)}
$$

holds for all a.e. $\xi \in \mathbb{R}$ and $q \in 2 \mathbb{Z}+1$. Consequently the set of all tight wavelet frames can not be dense in $L^{2}(\mathbb{R})$. Similar results hold for tight frames induced by group representations (c.f. Example 2 in [22]).

\section{The Bessel Vector Spaces}

Let $\mathcal{U}$ be a unitary system on $H$ and $\mathcal{B}_{\mathcal{U}}$ be the set of all the Bessel vectors for $\mathcal{U}$. It is easy from the definition of Bessel sequences to prove that $\mathcal{B}_{\mathcal{U}}$ is a linear subspace of $H$. Since the mapping from $\mathcal{B}_{\mathcal{U}}$ to $B\left(H, \ell^{2}(\mathcal{U})\right)$ defined by $x \rightarrow \Theta_{x}$ is linear and injective, we can identify $\mathcal{B}_{\mathcal{U}}$ with the space of all the analysis operators $\left\{\Theta_{x}: x \in \mathcal{B}_{\mathcal{U}}\right\}$ which is also a linear space. So we have two natural norms on $\mathcal{B}_{\mathcal{U}}$ : The Hilbert space norm $\|x\|$ and the operator norm $\left\|\Theta_{x}\right\|$. Let $\left\{\chi_{U}: U \in \mathcal{U}\right\}$ be the standard orthonormal basis for $\ell^{2}(\mathcal{U})$. Then $\Theta_{x}^{*} \chi_{U}=U x$ for all $U \in \mathcal{U}$. So we always have

$$
\|x\|=\left\|\Theta_{x}^{*} \chi_{I}\right\| \leq\left\|\Theta_{x}^{*}\right\|=\left\|\Theta_{x}\right\| .
$$

In general, $\mathcal{B}_{\mathcal{U}}$ is not necessarily closed with the Hilbert space norm. But the following proposition shows that it is a Banach space with the operator norm.

Proposition 3.1. The linear space $\mathcal{B}_{\mathcal{U}}$ is a Banach space with the operator norm, and $\left\{\Theta_{x}: x \in \mathcal{B}_{\mathcal{U}}\right\}$ is a closed subspace of $B\left(H, \ell^{2}(\mathcal{U})\right)$.

Proof. Let $\left\{\Theta_{x_{n}}\right\}$ be a Cauchy sequence. Then there exists a bounded linear operator $T \in B\left(H, \ell^{2}(\mathcal{U})\right)$ such that $\left\|\Theta_{x_{n}}-T\right\| \rightarrow 0$. Thus we have $\left\|\Theta_{x_{n}}^{*}-T^{*}\right\| \rightarrow 0$, in particular we have

$$
\left\|x_{n}-T^{*} \chi_{I}\right\|=\left\|\Theta_{x_{n}}^{*} \chi_{I}-T^{*} \chi_{I}\right\| \rightarrow 0 .
$$


So it suffices to check that $x$ is Bessel and $T=\Theta_{x}$ with $x=T^{*} \chi_{I}$. In fact, for any $y \in H$ and any finite subset $\Lambda$ of $\mathcal{U}$, we have

$$
\begin{aligned}
\sum_{U \in \Lambda}|\langle y, U x\rangle|^{2} & =\lim _{n \rightarrow \infty} \sum_{U \in \Lambda}\left|\left\langle y, U x_{n}\right\rangle\right|^{2} \\
& \leq \limsup _{n \rightarrow \infty} \sum_{U \in \mathcal{U}}\left|\left\langle y, U x_{n}\right\rangle\right|^{2} \\
& =\limsup _{n \rightarrow \infty}\left\|\Theta_{x_{n}}(y)\right\|^{2} \\
& \leq \limsup _{n \rightarrow \infty}\left\|\Theta_{x_{n}}\right\|^{2}\|y\|^{2} \leq K\|y\|^{2},
\end{aligned}
$$

where $K=\sup \left\{\left|\Theta_{x_{n}}\right|^{2}: n \in \mathbb{N}\right\}<\infty$. Thus $x$ is Bessel. Finally we show that $T=\Theta_{x}$. In fact, for any $U \in \mathcal{U}$ we have $\lim _{n \rightarrow \infty}\left\|\Theta_{x_{n}}^{*} \chi_{U}-T^{*} \chi_{U}\right\|=0$. On the other hand, since $\Theta_{x_{n}}^{*} \chi_{U}=U x_{n}$ and $\Theta_{x} \chi_{U}=U x$, we also have

$$
\lim _{n \rightarrow \infty}\left\|\Theta_{x_{n}}^{*} \chi_{U}-\Theta_{x}^{*} \chi_{U}\right\|=\lim _{n \rightarrow \infty}\left\|U x_{n}-U x\right\|=\lim _{n \rightarrow \infty}\left\|x_{n}-x\right\|=0 .
$$

Thus $T^{*} \chi_{U}=\Theta_{x}^{*} \chi_{U}$ for all $U \in \mathcal{U}$. Therefore we get $\Theta_{x}=T$, as claimed.

Note that $\left\|\Theta_{x}\right\|^{2}$ is the Bessel bound for $x$. So for the rest of the paper we will use $\|x\|_{o}$ to denote $\left\|\Theta_{x}\right\|$, and sometimes we refer it as the Bessel bound norm of $x$. Thus Proposition 3.1 states that the space $\left(\mathcal{B}_{\mathcal{U}},\|\cdot\|_{o}\right)$ is a Banach space. It is also an operator space with the set of matrix norms induced from the set of matrix norms of the operator space $\left\{\Theta_{x}: x \in \mathcal{B}_{\pi}\right\}$ considered as a closed subspace of $B\left(H, \ell^{2}(\mathcal{U})\right)$. We refer to [35] for more basic information about operator spaces.

In the case that $\mathcal{U}$ has a complete wandering vector $\psi$ (e.g. the wavelet unitary system $\left.\mathcal{U}_{D, T}\right)$, we use $\mathcal{C}_{\psi}(\mathcal{U})$ to denote the local commutant at $\psi$, which is defined by

$$
\mathcal{C}_{\psi}(\mathcal{U})=\{T \in B(H): T U \psi=U T \psi, \forall U \in \mathcal{U}\} .
$$

The we can also identify $\mathcal{B}_{\mathcal{U}}$ with $\mathcal{C}_{\psi}(\mathcal{U})$ by the following lemma:

Lemma 3.2. Assume that $\psi$ is a (complete) wandering vector for $\mathcal{U}$. Then $\eta$ is a Bessel vector for $\mathcal{U}$ if and only if there exists $B \in \mathcal{C}_{\psi}(\mathcal{U})$ such that $\eta=B \psi$. Moreover, this operator $B$ is unique, and $\|\eta\|_{o}=\|B\|$.

For self containment we give a short proof.

Proof. For each $\eta \in \mathcal{B}_{\mathcal{U}}$, we define $B_{\eta}$ by $B_{\eta} U \psi=U \eta$ for every $U \in \mathcal{U}$. Then we have that $B_{\eta} \in \mathcal{C}_{\psi}(\mathcal{U})$ and $B_{\eta} \psi=\eta$. Since $\{U \eta\}_{U \in \mathcal{U}}$ is an orthonormal basis, we have that $\left\|B_{\eta}\right\|=\left\|\Theta_{\eta}^{*}\right\|=\left\|\Theta_{\eta}\right\|=\|\eta\|_{o}$. For uniqueness, assume that $B^{\prime} \in \mathcal{C}_{\psi}(\mathcal{U})$ such that $B^{\prime} \psi=\eta$. Then we have for every $U \in U, B^{\prime} U \psi=U B^{\prime} \psi=U B_{\eta} \psi=B_{\eta} U \psi$. Thus $B^{\prime}=B_{\eta}$, as claimed.

\section{Bessel generator multipliers}

4.1. Examples of Bessel generator multipliers. A bounded linear operator $A$ on $H$ is a Bessel generator multiplier for $\mathcal{U}$ if it maps every Bessel vector of $\mathcal{U}$ to a Bessel vector of $\mathcal{U}$. In other words, a Bessel generator multiplier is a bounded linear operator $A$ such that 
$\mathcal{B}_{\mathcal{U}}$ is invariant under $A$. Here are some easy examples of of Bessel generator multipliers for a general unitary system.

Example 4.1. Let $\mathcal{U}$ be a unitary system on $H$.

(i) If $A$ commutes with $\mathcal{U}$, then $A$ is Bessel generator multiplier.

(ii) Let $A=\xi \otimes y$ be such that $\xi$ is a Bessel vector of $\mathcal{U}$. Then $A \eta=\langle\eta, y\rangle \xi$ is also a Bessel vector of $\mathcal{U}$ and hence $A$ is a Bessel generator multiplier

For a general unitary system it is very hard to have a good characterization for its Bessel generator multipliers. In this section we examine several interesting classes of Bessel generator multipliers. We consider a special case of unitary systems when $\mathcal{U}=\mathcal{U}_{1} \mathcal{U}_{0}$, where $\mathcal{U}_{0}$ is a countable group (but not necessarily abelian) and $\mathcal{U}_{1} \cap U_{0}=\{I\}$. These include the group systems, the wavelet systems and the Gabor systems (for the Gabor systems we refer to any standard literature for the definitions). In many cases we also require $\mathcal{U}_{1}$ to be a countable group. For a subset $\mathcal{A}$ of the algebra $B(H)$ of all bounded linear operators on $H$, we will use $w^{*}(\mathcal{A})$ to denote the von Neumann algebra generated by $\mathcal{A}$, and let $\mathcal{A}^{\prime}=\{S \in B(H): A S=S A, \forall S \in \mathcal{A}\}$ denote the commutant of $\mathcal{A}$. Clearly, the set of all Bessel generator multipliers for a unitary system $\mathcal{U}$ is a subalgebra of $B(H)$ with respect to the operator multiplication, and by Example 4.1 every operator in $\mathcal{U}^{\prime}$ is a Bessel generator multiplier.

Proposition 4.2. Let $\mathcal{U}=\mathcal{U}_{1} \mathcal{U}_{0}$ be a unitary system such that $\mathcal{U}_{0}$ is a countable group and $\mathcal{U}_{1} \cap U_{0}=\{I\}$. If $A \in w^{*}\left(\mathcal{U}_{0}\right) \cap \mathcal{U}_{0}^{\prime}$, then $A$ is a Bessel generator multiplier for $\mathcal{U}$.

Proof. Let $\psi$ be a Bessel vector for $\mathcal{U}$, let $S_{\psi} f=\sum_{U_{0} \in \mathcal{U}_{0}}\left\langle f, U_{0} \psi\right\rangle U_{0} \psi$. Then $S_{\psi} \in \mathcal{U}_{0}^{\prime}$. Moreover, since $A \in \mathcal{U}_{0}^{\prime}$, we get

$$
\begin{aligned}
S_{A \psi} f & =\sum_{U_{0} \in \mathcal{U}_{0}}\left\langle f, U_{0} A \psi\right\rangle U_{0} A \psi \\
& =A \sum_{U_{0} \in \mathcal{U}_{0}}\left\langle A^{*} f, U_{0} \psi\right\rangle U_{0} \psi \\
& =A S_{\psi} A^{*} f .
\end{aligned}
$$

Thus we have $S_{A \psi}=A S_{\psi} A^{*}$. Let $K=\|A\|^{2}$. Then $A A^{*} \leq K I$. By the assumption that $A \in w^{*}\left(\mathcal{U}_{0}\right)$ and the fact that $S_{\psi} \in \mathcal{U}_{0}^{\prime}$, we obtain $S_{\psi}^{1 / 2} A=A S_{\psi}^{1 / 2}$. Thus we have

$$
S_{A \psi}=A S_{\psi} A^{*}=S_{\psi}^{1 / 2} A A^{*} S_{\psi}^{1 / 2} \leq K S_{\psi}
$$


and so $U S_{A \psi} U^{*} \leq K U S_{\psi} U^{*}$ for any bounded operator $U$. This implies that

$$
\begin{aligned}
\sum_{U \in \mathcal{U}}(U A \psi) \otimes(U A \psi) & =\sum_{U_{1} \in \mathcal{U}_{1}, U_{0} \in \mathcal{U}_{0}}\left(U_{1} U_{0} A \psi\right) \otimes\left(U_{1} U_{0} A \psi\right) \\
& =\sum_{U_{1} \in \mathcal{U}_{1}} U_{1} S_{A \psi} U_{1}^{*} \\
& \leq K \sum_{U_{1} \in \mathcal{U}_{1}} U_{1} S_{\psi} U_{1}^{*} \\
& =K \sum_{U_{1} \in \mathcal{U}_{1}, U_{0} \in \mathcal{U}_{0}}\left(U_{1} U_{0} \psi\right) \otimes\left(U_{1} U_{0} \psi\right) \\
& =K \sum_{U \in \mathcal{U}}(U \psi) \otimes(U \psi) .
\end{aligned}
$$

Since $\psi$ is Bessel, we have that $\sum_{U \in \mathcal{U}}(U \psi) \otimes(U \psi)$ is weakly convergent to a positive bounded linear operator on $H$, and so $\sum_{U \in \mathcal{U}}(U A \psi) \otimes(U A \psi)$ is also weakly convergent to a positive bounded linear operator. Therefore $A \psi$ is a Bessel vector for $\mathcal{U}$, which implies that $A$ is a Bessel vector multiplier, as claimed.

Corollary 4.3. Let $\mathcal{U}=\mathcal{U}_{1} \mathcal{U}_{0}$ be a unitary system such that $\mathcal{U}_{0}$ is a group and $\mathcal{U}_{1} \cap U_{0}=\{I\}$. Assume that $\mathcal{U}_{0}$ is abelian. Then every operator in $w^{*}\left(\mathcal{U}_{0}\right)$ is a Bessel generator multiplier for $\mathcal{U}$.

Proposition 4.4. Let $\mathcal{U}=\mathcal{U}_{1} \mathcal{U}_{0}$ be a unitary system such that $\mathcal{U}_{0}$ is a group and $\mathcal{U}_{1} \cap U_{0}=$ $\{I\}$. Assume that $A \in \mathcal{U}_{0}^{\prime}$ is invertible and that $U_{1}^{*} A^{-1} U_{1} A \in w^{*}\left(\mathcal{U}_{0}\right)$ for every $U_{1} \in \mathcal{U}_{1}$, then $A$ is a Bessel generator multiplier for $\mathcal{U}$.

Proof. Let $\psi$ be a Bessel vector for $\mathcal{U}$. Fix $U_{1} \in \mathcal{U}_{1}$, let $B=U_{1}^{*} A^{-1} U_{1} A$ and let $S_{\psi}$ be defined as in the proof of Proposition 4.2. Since $S_{\psi} \in \mathcal{U}_{0}^{\prime}$, we have $B S^{\frac{1}{2}}=S^{\frac{1}{2}} B$. Thus we get

$$
B S_{\psi} B^{*}=S^{\frac{1}{2}}\left(B B^{*}\right) S^{\frac{1}{2}} \leq\|B\|^{2} \cdot S_{\psi} \leq K \cdot S_{\psi},
$$

where $K=\left(\left\|A^{-1}\right\| \cdot\|A\|\right)^{2}$ On the other hand we also have

$$
B S_{\psi} B^{*}=\left(U_{1}^{*} A^{-1} U_{1} A\right) S_{\psi}\left(A^{*} U_{1}^{*}\left(A^{-1}\right)^{*} U_{1}\right)=U_{1}^{*} A^{-1} U_{1} S_{A \psi} U_{1}^{*}\left(A^{-1}\right)^{*} U_{1}
$$

Therefore we get

$$
U_{1}^{*} A^{-1} U_{1} S_{A \psi} U_{1}^{*}\left(A^{-1}\right)^{*} U_{1} \leq K \cdot S_{\psi}
$$

which implies that

$$
U_{1} S_{A \psi} U_{1}^{*} \leq K\left(A^{-1}\right)^{*}\left[U_{1} S_{\psi} U_{1}^{*}\right] A^{-1}
$$

holds for every $U_{1} \in \mathcal{U}_{1}$. Since $\sum_{U_{1} \in \mathcal{U}_{1}} U_{1} S_{\psi} U_{1}^{*}$ is weakly convergent to a positive bounded linear operator, we have that $\sum_{U_{1} \in \mathcal{U}_{1}} U_{1} S_{A \psi} U_{1}^{*}$ is also weakly convergent to a positive bounded linear operator. Hence $A \psi$ is a Bessel vector for $\mathcal{U}$. 
4.2. The Bessel generator multiplier algebra. Let $\mathcal{M}_{\mathcal{U}}$ be the set of all Bessel generator multipliers for a unitary system $\mathcal{U}$. Then clearly, $\mathcal{M}_{\mathcal{U}}$ is a subalgebra of $B(H)$, and we will call it the Bessel generator multiplier algebra for $\mathcal{U}$. In general $\mathcal{M}_{\mathcal{U}}$ is not a closed subalgebra of $B(H)$, and in fact in many cases it is usually a very large dense subalgebra of $B(H)$ (see Proposition 4.6). Here we will introduce a natural quantity associated with the Bessel bounds for each operator in $\mathcal{M}_{\mathcal{U}}$. For any Bessel vector $\eta$ of $\mathcal{U}$, we will use $K_{\eta}$ to denote the optimal (minimal) Bessel bound of $\eta$. For $A \in \mathcal{M U}_{\mathcal{U}}$, we define the Bessel norm of $A$ by

$$
\|A\|_{b}=\sup \left\{\sqrt{K_{A \eta}}: K_{\eta} \leq 1, \eta \in \mathcal{B}_{\mathcal{U}}\right\}
$$

We will show that the Bessel norm is indeed a norm for the Bessel multiplier algebra, and we also compare the two norms for special classes of Bessel generator multipliers.

We already known that $K_{\xi}=\left\|\Theta_{\xi}\right\|^{2}=\|x\|_{o}^{2}$. Therefore we have

$$
\|A\|_{b}=\sup \left\{\|A \eta\|_{o}: \eta \in \mathcal{B}_{\mathcal{U}},\|\eta\|_{o}=1\right\} .
$$

Thus, the Bessel norm $\|\cdot\|_{b}$ will be indeed an norm if we could prove that $A:\left(\mathcal{B}_{\mathcal{U}},\|\cdot\|_{o}\right) \rightarrow$ $\left(\mathcal{B}_{\mathcal{U}},\|\cdot\|_{o}\right)$ is a bounded linear operator. Indeed this can be proven with the closed graph theorem.

Theorem 4.5. The Bessel norm $\|\cdot\|_{b}$ is an norm on $\mathcal{M}_{\mathcal{U}}$. Moreover, $\left(\mathcal{M}_{\mathcal{U}},\|\cdot\|_{b}\right) \cap\{A \in$ $B(H):\|A\| \leq 1\}$ is a closed subset of $B\left(\mathcal{B}_{\mathcal{U}},\|\cdot\|_{o}\right)$.

Proof. Let $A$ be a Bessel generator multiplier. Then it is a linear operator on $\mathcal{B}_{\mathcal{U}}$. Since $\|A\|_{b}=\sup \left\{\|A \eta\|_{o}: \eta \in \mathcal{B}_{\mathcal{U}},\|\eta\|_{o}=1\right\}$. We only need to show that $A$ is bounded with the norm $\|\cdot\|_{o}$ equipped on $\mathcal{B}_{\mathcal{U}}$.

Now let $x_{n}, x, y \in \mathcal{B}_{\mathcal{U}}$ be such that $\left\|x_{n}-x\right\|_{o} \rightarrow 0$ and $\left\|A x_{n}-y\right\|_{o} \rightarrow 0$. We need to show that $y=A x$. In fact, from the proof of Lemma 3.1, we must have $\left\|x_{n}-x\right\| \rightarrow 0$ and $\left\|A x_{n}-y\right\| \rightarrow 0$. Thus we have $y=A x$ since $A$ is a bounded linear operator on $H$. Therefore by the closed graph theorem we get that $A$ is bounded with the norm $\|\cdot\|_{o}$ equipped on $\mathcal{B}_{\mathcal{U}}$.

For the moreover part, suppose that $A_{n} \in \mathcal{M}_{\mathcal{U}}$ and $A \in B\left(\mathcal{B}_{\mathcal{U}},\|\cdot\|_{o}\right)$ such that $\| A_{n}-$ $A \|_{b} \rightarrow 0$ with $\left\|A_{n}\right\| \leq 1$. We will prove that $A$ is a restriction of a bounded linear operator on $H$, and hence $A \in \mathcal{M}_{\mathcal{U}}$. It suffices to show that $A \in B\left(\mathcal{B}_{\mathcal{U}},\|\cdot\|\right)$. In fact, from $\left\|A_{n}-A\right\|_{b} \rightarrow 0$, we get

$$
\left\|A_{n} x-A x\right\| \leq\left\|A_{n}-A\right\|_{b} \cdot\|x\|_{0} \rightarrow 0
$$

for every $x \in \mathcal{B}_{\mathcal{U}}$. This implies that

$$
\|A x\| \leq \sup \left\{\left\|A_{n} x\right\|: n \in \mathbb{N}\right\} \leq \sup \left\{\left\|A_{n}\right\| \cdot\|x\|: n \in \mathbb{N}\right\} \leq\|x\|
$$

for every $x \in \mathcal{B}_{\mathcal{U}}$. So $A$ can be extended to a bounded linear operator, say $\tilde{A}$, on $H$ with $\|\tilde{A}\| \leq 1$. Thus $\left(\mathcal{M}_{\mathcal{U}},\|\cdot\|_{b}\right) \cap\{A \in B(H):\|A\| \leq 1\}$ is a closed subset of $B\left(\mathcal{B}_{\mathcal{U}},\|\cdot\|_{o}\right)$.

In general $\left(\mathcal{M}_{\mathcal{U}},\|\cdot\|_{b}\right)$ is not complete (see Proposition 4.19). For operator norm closure we have the following: 
Proposition 4.6. Assume that $\mathcal{B}_{\mathcal{U}}$ is dense in $H$. Then the closure of $\mathcal{M}_{\mathcal{U}}$ in the usual operator norm contains all the compact operators, and hence $\mathcal{M}_{\mathcal{U}}$ is dense in $B(H)$ in the strong operator topology. Therefore, if $\mathcal{B}_{\mathcal{U}} \neq H$, then $\mathcal{M}_{\mathcal{U}}$ is not closed in the operator norm on $B(H)$.

Proof. Given $x, y \in H$. Since $\mathcal{B}_{\mathcal{U}}$ is dense in $H$, there exists a sequence $\left\{\eta_{n}\right\}$ from $\mathcal{B}_{\mathcal{U}}$ such that $\left\|\eta_{n}-x\right\| \rightarrow 0$. Let $A_{n}=\eta_{n} \otimes y$ and $A=x \otimes y$. Then $\left\|A_{n}-A\right\| \rightarrow 0$ and $A_{n} \in \mathcal{M}_{\mathcal{U}}$. Thus the operator norm closure of $\mathcal{M}_{\mathcal{U}}$ contains all the compact operators. Hence, $\mathcal{M}_{\mathcal{U}}$ is dense in $B(H)$ in the strong operator topology. In the case that $\mathcal{B}_{\mathcal{U}} \neq H$, let $x \notin \mathcal{B}_{\mathcal{U}}$ and $y \neq 0$. Then from the above argument $A=x \otimes y$ is in the operator norm closure of $\mathcal{M}_{\mathcal{U}}$, but clearly $A \notin \mathcal{M}_{\mathcal{U}}$. Thus $\mathcal{M}_{\mathcal{U}}$ is not closed in the operator norm on $B(H)$.

Proposition 4.7. (a) If $A \in \mathcal{U}^{\prime}$, then $\|A\|_{b} \leq\|A\|$. Moreover, if in addition assume that $\mathcal{U}$ admits a Parseval frame vector, then we have $\|A\|_{b}=\|A\|$.

(b) If $A \in w^{*}\left(\mathcal{U}_{0}\right) \cap \mathcal{U}_{0}^{\prime}$, we have $\|A\|_{b} \leq\|A\|$.

(c) If $A$ is a multiplier satisfying the condition in Proposition 4.4, then $\|A\|_{b} \leq\|A\|$. $\left\|A^{-1}\right\|^{2}$.

Proof. (a) Let $x \in \mathcal{B}_{\mathcal{U}}$. Then

$$
\|A x\|_{o}=\left\|\Theta_{A x}\right\|=\left\|\Theta_{x} A^{*}\right\| \leq\left\|A^{*}\right\| \cdot\left\|\Theta_{x}\right\|=\|A\| \cdot\|x\|_{o} .
$$

Thus $\|A\|_{b} \leq\|A\|$. For the in moreover part, let $] \xi$ be a Parseval frame vector. Then $\Theta_{\xi}$ is an isometry and $\|\xi\|_{0}=1$. Thus

$$
\|A\|_{b} \geq\|A \xi\|_{o}=\left\|\Theta_{A \xi}\right\|=\left\|\Theta_{\xi} A^{*}\right\|=\left\|A^{*}\right\|=\|A\|,
$$

and so we have the equality.

(b) Follows from the proof of Proposition 4.2.

(c) Follows from the proof of Proposition 4.4.

4.3. The Wavelet Unitary System. We show that for the wavelet unitary system, any of the following three cases could happen: (i) $\|A\|_{b}=\|A\|$; (ii) $\|A\|_{b}>\|A\|$; and (iii) $\|A\|_{b}<\|A\|$. Recall that the wavelet unitary system is the unitary system $\mathcal{U}_{D, T}:=\left\{D^{n} T^{\ell}\right.$ : $n, \ell \in \mathbb{Z}\}$, where $D$ is the dilation operator and $T$ is the translation unitary operator defined by

$$
(D f)(x)=\sqrt{2} f(2 x), \quad(T f)(x)=f(x-1), \quad f \in L^{2}(\mathbb{R}) .
$$

The Fourier transform we will use here is the normalized one:

$$
(\mathcal{F} f)(\xi)=\hat{f}(\xi)=\frac{1}{\sqrt{2 \pi}} \int_{\mathbb{R}} f(x) e^{-i x \xi} d x
$$

for all $f \in L^{1}(\mathbb{R})$ and then extend it unitarily to $L^{2}(\mathbb{R})$. With this definition we have $\hat{D}=\mathcal{F} D \mathcal{F}^{-1}=D^{-1}$ and $\hat{T}=\mathcal{F} T \mathcal{F}^{-1}=M_{e^{-i \xi}}$, where $M_{e^{-i \xi}}$ is the multiplication operator by $e^{-i \xi}$. It is known (cf. [8]) that the commutant of $\{\hat{D}, \hat{T}\}$ is $\left\{M_{h}: h \in L^{\infty}(\mathbb{R}), h(2 \xi)=\right.$ $h(\xi)$, a. e., $\xi \in \mathbb{R}\}$. A Parseval frame set is a measurable subset $F$ of $\mathbb{R}$ such that $\left\{2^{n} F: n \in\right.$ $\mathbb{Z}\}$ is a partition of $\mathbb{R}$ (modulo measure zero sets) and $\{F+2 n \pi: n \in \mathbb{Z}\}$ is disjoint (modulo measure zero sets). Equivalently, $F$ is a Parseval frame set if and only if $\left\{\hat{D}^{n} \hat{T}^{\ell}\left(\frac{1}{\sqrt{2 \pi}} \chi_{F}\right)\right.$ : $n, \ell \in \mathbb{Z}\}$ is a Parseval frame for $L^{2}(\mathbb{R})$ (c.f. [20]). By Proposition 4.7 (a) we have that 
$\|A\|_{b}=\|A\|$ if $A \in\{D, T\}^{\prime}$. So we only need to show that the other two cases could happen for the wavelet unitary system.

Example 4.8. There exists a Bessel generator multiplier for the wavelet unitary system such that $\|A\|_{b}>\|A\|$.

Proof. Let $A=\psi \otimes \varphi$ be such that $\psi$ is a Bessel wavelet and $\psi \in L^{2}(\mathbb{R})$. Then, for evry $f \in L^{2}(\mathbb{R}), A f=\langle f, \varphi\rangle \psi$ is also a Bessel wavelet and hence $A$ is a Bessel wavelet multiplier with Bessel bound $K_{A f}=|\langle f, \varphi\rangle|^{2} K_{\psi}$.

Now let $\hat{\psi}(\xi)=\frac{1}{\sqrt{2 \pi}} \chi_{[-\pi,-\pi / 2) \cup[\pi / 2, \pi)}$ and $\left.\hat{\varphi}(\xi)=\frac{1}{\sqrt{2 \pi}} \chi_{[-2 \pi,-\pi) \cup[\pi, 2 \pi)}\right)$. Then $\psi$ is a Bessel vector such that $\|\psi\|<\|\psi\|_{o}=1$ and $\varphi$ is an orthonormal wavelet such that $\|\varphi\|=\|\varphi\|_{o}=1$. Hence we get

$$
\|A\|_{b}^{2} \geq\left\|\Theta_{A \varphi}\right\|^{2}=|\langle\varphi, \varphi\rangle|^{2} K_{\psi}=K_{\psi}=\|\psi\|_{o}^{2}>\|\psi\|^{2}=\|A\|^{2}
$$

since $\|A\|=\|\varphi\|\|\psi\|=\|\psi\|$, and so we have $\|A\|_{b}>\|A\|$.

Example 4.9. There exists a Bessel generator multiplier for the wavelet unitary system such that $\|A\|_{b}<\|A\|$.

Proof. Let $E=\left[\frac{\pi}{4}, \frac{\pi}{2}\right]$, and define $A$ by $(\hat{A} f)(\xi)=\chi_{2 E}(\xi) f\left(\frac{\xi}{2}\right)$. We prove that $\|A\|=\sqrt{2}$ and $\|A\|_{b}=1$, and consequently we get $\|A\|_{b}<\|A\|$.

Assume that $f \in L^{2}(\mathbb{R})$ be such that $\operatorname{supp}(\hat{f}) \subseteq E$. Then

$$
\|A f\|^{2}=\int_{2 E}\left|\chi_{2 E}(\xi) \hat{f}\left(\frac{\xi}{2}\right)\right|^{2} d \xi=2 \int_{E}|\hat{f}(t)|^{2} d t=2\|\hat{f}\|^{2}=2\|\| f \|^{2} .
$$

Thus $\|A\|=\sqrt{2}$.

Next we show that $\|A\|_{b}=1$. We will need two facts.

Fact 1: Let $g$ be a Bessel wavelet for the wavelet system such that $\operatorname{supp}(\hat{g})$ is contained in a Parseval frame set $F$. Then we claim that $\|g\|_{o}=\sqrt{2 \pi}\|\hat{g}\|_{\infty}$. In fact, since $F$ is Parseval frame set, we have that $\left\{2^{n} F: n \in \mathbb{Z}\right\}$ is a disjoint partition of $\mathbb{R}$. So we can extend the restriction of $\hat{g}$ on $F$ to a 2 -dilation function $\hat{h}$ (i.e. $\hat{h}(2 \xi)=\hat{h}(\xi)$, a.e. $\xi \in \mathbb{R}$ ). Then $\|\hat{g}\|_{\infty}=\|\hat{h}\|_{\infty}$, and the multiplication operator $M_{\hat{h}}$ commutes with both operators $\hat{D}$ and $\hat{T}$. Let $\hat{\psi}=\frac{1}{\sqrt{2 \pi}} \chi_{F}$. Then $\left\{\psi_{k, j}: k, j \in \mathbb{Z}\right\}$ is a Parseval frame for $L^{2}(\mathbb{R})$, and so $\sum_{k, j \in \mathbb{Z}} \hat{D}^{k} \hat{T}^{j} \hat{\psi} \otimes \hat{D}^{k} \hat{T}^{j} \hat{\psi}=I$. Note that $\hat{g} \hat{\psi}=\hat{h} \hat{\psi}$. So we get

$$
\begin{aligned}
S_{\hat{g}} & =\sum_{k, j \in \mathbb{Z}} \hat{D}^{k} \hat{T}^{j} \hat{g} \otimes \hat{D}^{k} \hat{T}^{j} \hat{g} \\
& =2 \pi \sum_{k, j \in \mathbb{Z}} \hat{D}^{k} \hat{T}^{j} M_{\hat{g}} \hat{\psi} \otimes \hat{D}^{k} \hat{T}^{j} M_{\hat{g}} \hat{\psi} \\
& =2 \pi \sum_{k, j \in \mathbb{Z}} \hat{D}^{k} \hat{T}^{j} M_{\hat{h}} \hat{\psi} \otimes \hat{D}^{k} \hat{T}^{j} M_{\hat{h}} \hat{\psi} \\
& =2 \pi M_{\hat{h}}\left(\sum_{k, j \in \mathbb{Z}} \hat{D}^{k} \hat{T}^{j} \hat{\psi} \otimes \hat{D}^{k} \hat{T}^{j} \hat{\psi}\right) M_{\hat{h}}^{*} \\
& =2 \pi M_{|\hat{h}|^{2}}
\end{aligned}
$$


So we have $\|g\|_{0}=\sqrt{\left\|S_{\hat{g}}\right\|}=\sqrt{2 \pi}\|\hat{h}\|_{\infty}=\sqrt{2 \pi}\|\hat{g}\|_{\infty}$ since $\|\hat{g}\|_{\infty}=\|\hat{h}\|_{\infty}$.

Fact 2: If $g$ is a Bessel wavelet, then $\sqrt{2 \pi}\|\hat{g}\|_{\infty} \leq\|g\|_{o}$. It is a well known fact [24] that for any compactly supported function $\hat{f}$, we have

$$
\int_{\mathbb{R}}|\hat{f}(\xi)|^{2} \sum_{k \in \mathbb{Z}}\left|\hat{g}\left(2^{k} \xi\right)\right|^{2} d \xi \leq \frac{1}{2 \pi} \sum_{k, j \in \mathbb{Z}}\left|\left\langle\hat{f}, \hat{D}^{k} \hat{T}^{j} \hat{g}\right\rangle\right|^{2}
$$

Therefore we get

$$
\int_{\mathbb{R}}|\hat{f}(\xi)|^{2} \sum_{k \in \mathbb{Z}}\left|\hat{g}\left(2^{k} \xi\right)\right|^{2} d \xi \leq \frac{1}{2 \pi}\|g\|_{o}^{2} \cdot\|\hat{f}\|^{2} .
$$

Thus $\sum_{k \in \mathbb{Z}}\left|\hat{g}\left(2^{k} \xi\right)\right|^{2} \leq \frac{1}{2 \pi}\|g\|_{o}^{2}$ for a.e. $\xi \in \mathbb{R}$, which implies that $\sqrt{2 \pi}\|\hat{g}\|_{\infty} \leq\|g\|_{o}$.

Now let $g$ be any Bessel wavelet for the wavelet system. Then $\operatorname{supp}(\hat{A} \hat{g}) \subseteq 2 E$. Since $2 E$ is a subset of the Shannon wavelet set $[-2 \pi,-\pi) \cup[\pi, 2 \pi)$, we get from Fact 1 that $\|A g\|_{o}=\sqrt{2 \pi}|| \hat{A} \hat{g}\left\|_{\infty} \leq \sqrt{2 \pi}\right\| \hat{g} \|_{\infty}$. However, by Fact 2, we also have $\sqrt{2 \pi}\|\hat{g}\|_{\infty} \leq\|g\|_{o}$. Therefore we obtain that $\|A g\|_{o} \leq\|g\|_{o}$ and hence $\|A\|_{b} \leq 1$. On the other hand, if $g$ be a Bessel wavelet for the wavelet system such that $\hat{g}(\xi)=\chi_{E}(\xi)$. Then we get $\|A g\|_{o}=$ $\sqrt{2 \pi}\|\hat{g}\|_{\infty}=\|g\|_{o}$. Thus $\|A\|_{b}=1$, as claimed.

A Bessel wavelet multiplier is called a Fourier Bessel wavelet multiplier if is of the form $\hat{A}=M_{h}$ for some $h \in L^{\infty}(\mathbb{R})$. Note that none of the Bessel wavelet multipliers in the last two example is a Fourier Bessel wavelet multiplier. For general Fourier Bessel wavelet multipliers we have the following:

Example 4.10. Assume that $h$ is a Fourier Bessel wavelet multiplier for the wavelet unitary system. Then $\|A\| \leq\|A\|_{b}$.

Proof. For any $\epsilon>0$, we can find measurable subset $E$ with positive measure such that $|h(\xi)| \geq\|h\|_{\infty}-\epsilon$ on $E$, and $E$ is both 2-dilation disjoint and $2 \pi$-translation disjoint. Let $\hat{\psi}=\frac{1}{\sqrt{2 \pi}} \chi_{E}$. Then $\psi$ is a Bessel vector with Bessel constant 1, i.e. $\|\psi\|_{o}=1$. Let $\varphi=A \psi$ (i.e. $\hat{\varphi}=h \hat{\psi}$ ). We calculate the Bessel bound of $\varphi$. Note that $\left\langle\hat{\varphi},(h \hat{\psi})_{j, k}\right\rangle=0$ if $j \neq 0$. So we have

$$
\begin{aligned}
\sum_{j, k \in \mathbb{Z}}\left|\left\langle\varphi,(h \psi)_{j, k}\right\rangle\right|^{2} & =\sum_{k \in \mathbb{Z}}\left|\left\langle\varphi,(h \psi)_{0, k}\right\rangle\right|^{2} \\
& =\|\varphi\|^{2}=\frac{1}{2 \pi} \int_{E}|h(\xi)|^{2} d \xi \\
& \geq\left(\|h\|_{\infty}-\epsilon\right)\|\varphi\|^{2} .
\end{aligned}
$$

This $\varphi$ has Bessel bound no less that $\|h\|_{\infty}-\epsilon$, which implies that $\|A\|_{b} \geq\|h\|_{\infty}-\epsilon$ for all small $\epsilon>0$. This implies that

$$
\|A\|_{b} \geq\|h\|_{\infty}=\|A\|,
$$

as claimed. 
We have the post the following open problem:

Problem A. Is it true that $\|A\|_{b}=\|A\|$ for all Fourier Bessel multipliers $A$ ?

We conjecture that answer is affirmative, and point out the following partial result.

Corollary 4.11. Assume that $h$ is a Fourier Bessel wavelet multiplier for the wavelet unitary system such that $\hat{h}$ is either $2 \pi$-translation or 2-dilation periodic, then for $A=\check{M}_{h}$ we have $\|A\|=\|A\|_{b}$.

Proof. From the proof of Proposition 2.1 in $[22]$ we get that $\|A\|_{b} \leq\|\hat{h}\|_{\infty}=\|A\|$. Thus the conclusion follows from Example 4.10.

4.4. The group unitary system case. In this subsection we discuss the case when the unitary system is the image of a projective unitary representation of a countable group. Recall (cf. [38]) that a projective unitary representation $\pi$ for a countable group $G$ is a mapping $g \rightarrow \pi(g)$ from $G$ into the group $U(H)$ of all the unitary operators on a separable Hilbert space $H$ such that $\pi(g) \pi(h)=\mu(g, h) \pi(g h)$ for all $g, h \in G$, where $\mu(g, h)$ is a scalar-valued function on $G \times G$ taking values in the circle group $\mathbb{T}$. This function $\mu(g, h)$ is then called a multiplier of $\pi$. In this case we also say that $\pi$ is a $\mu$-projective unitary representation. It is clear from the definition that we have

(i) $\mu\left(g_{1}, g_{2} g_{3}\right) \mu\left(g_{2}, g_{3}\right)=\mu\left(g_{1} g_{2}, g_{3}\right) \mu\left(g_{1}, g_{2}\right)$ for all $g_{1}, g_{2}, g_{3} \in G$,

(ii) $\mu(g, e)=\mu(e, g)=1$ for all $g \in G$, where $e$ denotes the group unit of $G$.

Any function $\mu: G \times G \rightarrow \mathbb{T}$ satisfying $(i)-(i i)$ above will be called a multiplier for $G$. It follows from $(i)$ and $(i i)$ that we also have

(iii) $\mu\left(g, g^{-1}\right)=\mu\left(g^{-1}, g\right)$ holds for all $g \in G$.

Examples of projective unitary representations include unitary group representations and Gabor representations in time-frequency analysis. In what follows we will consider the unitary system $\pi(G)$, and use $\mathcal{B}_{\pi}$ to denote the set of all Bessel vectors for $\pi(G)$, where $\pi$ is a projective unitary representation of $G$.

Lemma 4.12. [9] Let $\pi$ be a projective representation of a countable group $G$ on a Hilbert space $H$ and $x$ is a Bessel vector for $\pi$. Then there exists $\xi \in M:=\overline{\operatorname{span}}\{\pi(g) x: g \in G\}$ such that $\{\pi(g) \xi\}_{g \in G}$ is a Parseval frame for $M$.

Lemma 4.13. [18] Assume that $\pi$ is a projective representation of a countable group $G$ on a Hilbert space $H$ and $x$ is a complete Parseval frame vector for $\pi$. Let $\eta \in H$. Then we have

(i) $\eta$ is a Bessel vector for $\pi$ if and only if there exists an operator $T \in w^{*}(\pi(G))$ such that $\eta=T \xi$.

(ii) $\eta$ is a complete frame vector for $\pi$ if and only if there exists an invertible operator $T \in w^{*}(\pi(G))$ such that $\eta=T \xi$.

(iii) $\eta$ is a complete Parseval frame vector for $\pi$ if and only if there exists an unitary operator $T \in w^{*}(\pi(G))$ such that $\eta=T \xi$.

Proposition 4.14. Let $\pi$ be a projective unitary representation of $G$. If $A \in w^{*}(\pi(G))$, then $A$ is a Bessel generator multiplier. 
Proof. Let $\xi$ be a Bessel vector for $\pi$. Then, by Lemma 4.12, there exists $\eta \in M:=$ $\overline{\operatorname{span}}\{\pi(g) \xi: g \in G\}$ such that $\{\pi(g) \eta: g \in G\}$ is a Parseval frame for $M$. Thus, from Lemma 4.13 that a vector $x \in M$ is a Bessel vector if and only if there exists $\left.B \in w^{*}(\pi(G))\right|_{M}$ such that $x=B \eta$. Let $\xi=T \eta$ with $\left.T \in w^{*}(\pi(G))\right|_{M}$. Then $A \xi=A T \eta$ with $A T \in$ $\left.w^{*}(\pi(G))\right|_{M}$ is also Bessel. Therefore $A$ is a Bessel generator multiplier.

Theorem 4.15. Let $\pi$ be a projective unitary representation of $G$ such that $\mathcal{B}_{\pi}$ is dense in $H$. Then

(i) $\|A\|_{b}=\|A\|$ if $A \in \pi(G)^{\prime}$

(ii) $\|A\|_{b} \leq\|A\|$ if $A \in w^{*}(\pi(G))$.

We remark that part (i) of this theorem is not the consequence of Proposition 4.7 since here we do not assume $\pi$ admits a Parseval frame vector for $H$.

In order to prove Theorem 4.15 we need the following lemma:

Lemma 4.16. Let $\pi$ be a projective unitary representation of $G$ such that its has a complete wandering vector $\psi$. Then $\|A\|_{b}=\|A\|$ when $A \in w^{*}(\pi(G))$.

Proof. Let $\sigma: w^{*}(\pi(G)) \rightarrow \pi(G)^{\prime}$ be the conjugate linear anti-automorphism such that $T \psi=\sigma(T) \psi$ for all $T \in w^{*}(\pi(G))$ (cf. [28]). Let $A \in w^{*}(\pi(G))$. Then

$$
\|A\|_{b} \geq\left\|\Theta_{A \psi}\right\|=\left\|\Theta_{\sigma(A) \psi}\right\|=\left\|\Theta_{\psi} \sigma(A)^{*}\right\|=\left\|\sigma(A)^{*}\right\|=\|A\|,
$$

where we use the isometry property of $\Theta_{\psi}$.

On the other hand, for any Bessel vector $x \in \mathcal{B}_{\pi}$, there exists $B \in \pi(G)^{\prime}$ such that $x=B \psi$. Thus we have

$$
\begin{aligned}
\|A x\|_{b} & =\left\|\Theta_{A x}\right\|=\left\|\Theta_{A B \psi}\right\|=\left\|\Theta_{B A \psi}\right\|=\left\|\Theta_{B \sigma(A) \psi}\right\| \\
& =\left\|\Theta_{\psi} \sigma(A)^{*} B^{*}\right\| \leq\left\|\sigma(A)^{*}\right\| \cdot\left\|\theta_{\psi}\right\| \cdot\left\|B^{*}\right\| \\
& =\|A\| \cdot\left\|\theta_{\psi}\right\| \cdot\|B\|=\|A\| \cdot\|B\| .
\end{aligned}
$$

Note that $\|x\|_{o}=\left\|\Theta_{x}\right\| \cdot\left\|\Theta_{B \psi}\right\|=\left\|B^{*}\right\|=\|B\|$. Thus we get

$$
\|A x\|_{b} \leq\|A\| \cdot\|x\|_{o}
$$

for all $x \in \mathcal{B}_{\pi}$. This implies that $\|A\|_{b} \leq\|A\|$, as claimed.

Lemma 4.17. Let $\pi$ be a $\mu$-projective unitary representation of $G$ on a Hilbert space $H$ such that its has a complete frame vector $\psi$. Then there exists a $\mu$-projective unitary representation $\sigma$ of $G$ on a Hilbert space $K$ such that the $\mu$-projective unitary representation of $\pi \oplus \sigma$ on $H \oplus K$ has complete wandering vector.

Corollary 4.18. Let $\pi$ be a projective unitary representation of $G$ such that its has a complete frame vector. Then $\|A\|_{b} \leq\|A\|$ when $A \in w^{*}(\pi(G))$.

Proof. By Lemma 4.17, we can dilate $\pi$ to $\pi \oplus \sigma$ on $H \oplus K$ for some Hilbert space $K$ such that $\pi \oplus \sigma$ has complete wandering vector. Let $\tilde{A} \in w^{*}(\pi(g) \oplus \sigma(g): g \in G)$ such that $\left.\tilde{A}\right|_{H}=A$ and $\|\tilde{A}\|=\|A\|$. By Lemma 4.16, we have $\|\tilde{A}\|_{b}=\|\tilde{A}\|$. Thus $\|A\|_{b} \leq\|\tilde{A}\|_{b}=$ $\|\tilde{A}\|=\|A\|$. 


\section{Proof of Theorem 4.15:}

(i) For any $\xi \in \mathcal{B}_{\pi}$, we have

$$
\sum_{g \in G}|<x, \pi(g) A g>|^{2}=\sum_{g \in G}\left|<A^{*} x, \pi(g) g>\right|^{2} \leq\left\|A^{*}\right\|^{2} K_{g}\|x\|, \quad x \in H
$$

where $K_{g}$ is Bessel bound of $g$. Thus we have $\|A\|_{b} \leq\|A\|$. So we only need to show that $\|A\|_{b} \geq\|A\|$. Since $\mathcal{B}_{\pi}$ is dense in $H$, for each $\epsilon>0$ we can find $x \in \mathcal{B}_{\pi}$ with $\|x\|=1$ and $\left\|A^{*} x\right\| \geq\|A\|-\epsilon$. Let $A^{*} x$, and $H_{x}=\left[\pi(G) A^{*} x\right]$. Then, by Lemma 4.12, there exists a $\xi \in H_{x}$ such that $\{\pi(g) \xi\}$ is a Parseval frame for $H_{y}$. So $\|\xi\|_{o}=1$, and

$$
\|A\|_{b} \geq\left\|\Theta_{A \xi}\right\|=\left\|\Theta_{\xi} A^{*}\right\| \geq\left\|\Theta_{\xi} A^{*} x\right\|=\left\|A^{*} x\right\| \geq\|A\|-\epsilon,
$$

where we use that $\Theta_{\xi}$ is an isometry when restricted to $H_{y}$ and $A^{*} x \in H_{y}$. Thus $\|A\|_{b} \geq\|A\|$ by letting $\epsilon \rightarrow 0$.

(ii) Let $x \in \mathcal{B}_{\pi}$ such that $\|x\|_{0} \leq 1$. Let $M=[\pi(G) x]$ and consider $\left.\pi\right|_{M}$. Then, by Corollary 4.18 , we get $\|\bar{A}\|_{b} \leq\|\bar{A}\|$. This implies that

$$
\|A x\|_{o}=\|\bar{A} x\|_{o} \leq\|\bar{A}\|_{b} \leq\|\bar{A}\| \leq\|A\| .
$$

Taking the sup over $\|x\|_{o} \leq 1$, we get

$$
\|A\|_{b} \leq\|A\|
$$

as claimed.

Problem B. Let $\pi$ be a projective unitary representation of $G$. Do we have $\|A\|_{b}=\|A\|$ for $A \in w^{*}(\pi(G))$ ? What about other Bessel generator multiplier?

Let $A$ be a Bessel generator multiplier for a general unitary system $\mathcal{U}$. Then, by Theorem 4.5 we have that $A$ is also a bounded linear mapping $\Phi_{A}$ from the operator space $\left(\mathcal{B}_{\mathcal{U}},\|\cdot\|_{o}\right)$ into itself. One of the most important concept in the theory of operator spaces is the completely bounded maps. Recall that a linear mapping $\Phi$ from an operator space $S$ to an operator space $V$ is called completely bounded if $\sup \left\{\left\|\Phi_{n}\right\|: n \geq 1\right\}<\infty$, where $\Phi_{n}$ defined by

$$
\Phi_{n}\left(\left[a_{i j}\right]\right)=\left[\Phi\left(a_{i j}\right]\right.
$$

on the operator space $M_{n} \otimes S$. A natural question about the Bessel vector space is to examine the completely boundedness of $\Phi_{A}$. We ask the following:

Problem C. Is $\Phi_{A}$ always completely bounded?

We examine a special case of this question. Assume that $\mathcal{U}$ admits a complete wandering vector, say $\psi$. Then, by Lemma 3.2 , we can identify $\left(\mathcal{B}_{\mathcal{U}},\|\cdot\|_{o}\right)$ with the local commutant operator space $\mathcal{C}_{\psi}(\mathcal{U})$. Therefore, if $A$ is a Bessel generator multiplier for $\mathcal{U}$, then induced mapping $\Phi_{A}$ is defined by $\Phi_{A}(S)=T$ where $T$ is the unique operator in $\mathcal{C}_{\psi}(\mathcal{U})$ such that $T \psi=A S \psi$. However, in general we don't have $T=A S$ since $A S$ may not be in $\mathcal{C}_{\psi}(\mathcal{U})$. In the case that $A \in \mathcal{U}^{\prime}$, then $A S \in \mathcal{C}_{\psi}(\mathcal{U})$ for every $S \in \mathcal{C}_{\psi}(\mathcal{U})$, and therefore $\Phi_{A}(S)=A S$, and so $\Phi$ is completely bounded. However, it remains open whether all the induced mapping $\Phi_{A}$ is completely bounded even when $\mathcal{U}$ admits a complete wandering vector. 
The second natural question is to ask that if every bounded linear mapping from the operator space $\left(\mathcal{B}_{\mathcal{U}},\|\cdot\|_{o}\right)$ to itself is induced by a Bessel generator multiplier from $B(H)$. The following result answers this question negatively.

Proposition 4.19. Let $\mathcal{U}$ be the wavelet unitary system. Then there exists a bounded linear mapping $\Phi$ from Bessel generator multiplier the operator space $\left(\mathcal{B}_{\mathcal{U}},\|\cdot\|_{o}\right)$ to itself such that is the limit of a Bessel generator multipliers in the Bessel norm. But $\Phi$ is unbounded with respect to the Hilbert space norm on $\mathcal{B}_{\mathcal{U}}$. In particular, the Bessel generator multiplier algebra $\left(\mathcal{M}_{\mathcal{U}},\|\cdot\|_{b}\right)$ is not complete.

Proof. Let $E_{n}=\left[\pi / 2^{n+1}, \pi / 2^{n}\right)$ for $n \geq 1$. Define $A_{n}$ by $\hat{A_{n}} f=\chi_{2^{n} E_{n}}(\xi) f\left(\frac{\xi}{2^{n}}\right)$. Then, as in the proof of Example 4.9, we get that $\left\|A_{n}\right\|=2^{n / 2}$, and $\left\|A_{n}\right\|_{b}=1$ for all $n \geq 1$. Now let $B_{n}=2^{-n / 4} A_{n}$. Then $\sum_{n=1}^{\infty} B_{n}$ converges in the $\|\cdot\|_{b}$ norm on $\left(\mathcal{B}_{\mathcal{U}},\|\cdot\|_{o}\right)$. Let $\Phi=\sum_{n=1}^{\infty} \hat{B}_{n}$.

We claim that there is no Bessel generator multiplier $A$ for $\mathcal{U}$ such that $\Phi_{A}=\Phi$, i.e, $\Phi \neq A$ on $\mathcal{B}_{\mathcal{U}}$ for all Bessel generator multiplier $A$ for $\mathcal{U}$. In order to prove this, it suffices to show that $\Phi$ is an unbounded linear operator with respect to the Hilbert space norm on $\left(\mathcal{B}_{\mathcal{U}}\right.$. In fact, let $f_{n}=\chi_{E_{n}}$. Since $\hat{B_{m}} f_{n}=0$ if $m \neq n$, we have $\Phi\left(f_{n}\right)=\hat{B_{n}} f_{n}$. So

$$
\left\|\Phi\left(f_{n}\right)\right\|^{2}=\left\|\hat{B}_{n} f_{n}\right\|^{2}=2^{-n / 2} \mu\left(2^{n} E_{n}\right)=2^{-n / 2}\left(\frac{\pi}{2}\right) .
$$

But $\left\|f_{n}\right\|^{2}=\mu\left(E_{n}\right)=\frac{\pi}{2^{n+1}}$. Thus $\left\|\Phi\left(f_{n} /\left\|f_{n}\right\|\right)\right\|^{2}=2^{n / 2} \rightarrow \infty$. Hence $\Phi$ is unbounded as claimed.

\section{REFERENCES}

[1] R. Balan, A study of Weyl-Heisenberg and wavelet frames, Ph. D. Thesis, Princeton University, 1998.

[2] M. Bownik, Connectivity and density in the set of framelets Math. Res. Lett., 14 (2007), 285-293.

[3] M. Bownik, The closure of the set of tight frame wavelets, Acta Appl. Math., 107 (2009), 195-201.

[4] X. Dai and Y. Diao, The path-connectivity of s-elementary tight frame wavelets, J. Appl. Funct. Anal., 2 (2007), no. 4, 309-316.

[5] X. Dai, Y. Diao and Q. Gu, Frame wavelets with frame set support in the frequency domain, Illinois J. Math., 48 (2004), no. 2, 539-558.

[6] X. Dai, Y. Diao, Q. Gu and D. Han, The $S$-elementary frame wavelets are path connected, Proc. Amer. Math. Soc., 132 (2004), no. 9, 2567-2575.

[7] X. Dai, Y. Diao, Q. Gu and D. Han, Frame wavelets in subspaces of $L^{2}\left(\mathbb{R}^{d}\right)$, Proc. Amer. Math. Soc., 130 (2002), no. 11, 3259-3267.

[8] X. Dai and D. Larson, Wandering vectors for unitary systems and orthogonal wavelets, Mem. Amer. Math. Soc., 134 (1998), no. 640.

[9] J. Gabardo and D. Han, Subspace Weyl-Heisenberg frames, J. Fourier Anal. Appl., 7 (2001), 419-433.

[10] J-P. Gabardo and D. Han, Aspects of Gabor analysis and operator algebras. Advances in Gabor analysis, 129-152, Appl. Numer. Harmon. Anal., Birkhuser Boston, Boston, MA, 2003

[11] J-P. Gabardo and D. Han, Frame representations for group-like unitary operator systems, J. $O p$ erator Theory , 49 (2003), 223-244. 
[12] J-P. Gabardo and D. Han, The uniqueness of the dual of Weyl-Heisenberg subspace frames, Appl. Comput. Harmon. Anal., 17 (2004), no. 2, 226-240.

[13] J-P. Gabardo, D. Han and D. Larson, Gabor frames and operator algebras, Wavelet Applications in Singnal and Image Analysis, Proc. SPIE., 4119 (2000), 337-345.

[14] G. Garrigós, Connectivity, homotopy degree, and other properties of $\alpha$-localized wavelets on $R$, Publ. Mat., 43 (1999), no. 1, 303-340.

[15] G. Garrigós, E. Hernández, H. vSikić and F. Soria, Further results on the connectivity of Parseval frame wavelets, Proc. Amer. Math. Soc., 134 (2006), no. 11, 3211-3221.

[16] G. Garrigós, E. Hernández, H. Sikić, F. Soria, G. Weiss and E. Wilson, Connectivity in the set of tight frame wavelets (TFW), Glas. Mat. Ser. III. 38(58) (2003), no. 1, 75-98.

[17] G. Garrigós and D. Speegle, Completeness in the set of wavelets, Proc. Amer. Math. Soc., 128 (2000), no. 4, 1157-1166.

[18] D. Han, Approximations for Gabor and wavelet frames, Trans. Amer. Math. Soc., 355 (2003), no. 8, 3329-3342.

[19] D. Han, Frame Representations and Parseval Duals with Applications to Gabor Frames, Trans. Amer. Math. Soc., 360(2008), 3307-3326.

[20] D. Han and D. Larson, Frames, bases and group parametrizations, Memoirs Amer. Math. Soc., 697 (2000).

[21] D. Han and D. Larson, Wandering vector multipliers for unitary groups, Trans. Amer. Math. Soc., $353(2001), 3347-3370$

[22] D. Han and D. Larson, On the orthogonality of frames and the density and connectivity of wavelet frames, Acta Appl. Math., 107 (2009), 211-222.

[23] D. Han, Q. Sun and W. Tang, Topological and geometric properties of refinable functions and MRA affine frames, preprint, 2006.

[24] E. Hernández, and G. Weiss, A First Course on Wavelets, CRC Press, 1996.

[25] E. Hernández, X. Wang and G. Weiss, Smoothing minimally supported frequency wavelets. I. J. Fourier Anal. Appl., 2 (1996), no. 4, 329-340.

[26] E. Hernández, X. Wang and G. Weiss, Smoothing minimally supported frequency wavelets. II., J. Fourier Anal. Appl., 3 (1997), no. 1, 23-41.

[27] G. Ji and K. Saito, On wandering vector multipliers for unitary groups, Proc. Amer. Math. Soc., 133 (2005), 3263-3269.

[28] R. Kadison and J. Ringrose, Fundamentals of the Theory of Operator Algebras, Vol. I and II, Academic Press, Inc. 1983 and 1985.

[29] D. Larson, Von Neumann algebras and wavelets. Operator algebras and applications (Samos, 1996), 267-312, NATO Adv. Sci. Inst. Ser. C Math. Phys. Sci., 495, Kluwer Acad. Publ., Dordrecht, 1997.

[30] D. Larson, Frames and wavelets from an operator-theoretic point of view. Operator algebras and operator theory (Shanghai, 1997), 201-218, Contemp. Math., 228, Amer. Math. Soc., Providence, RI, 1998.

[31] D. Larson, Unitary systems and wavelet sets. Wavelet analysis and applications, 143-171, Appl. Numer. Harmon. Anal., Birkhuser Basel, 2007.

[32] D. Larson, Unitary systems, wavelet sets, and operator-theoretic interpolation of wavelets and frames, Gabor and Wavelet Frames, World Scientific (2007), 166-214.

[33] R. Liang, Wavelets, their phases, multipliers and connectivity, Ph. D. Thesis, University of North Carolina-Charlotte, 1998.

[34] M. Paluszynski, H. Sikic, G. Weiss and S. Xiao, Tight frame wavelets, their dimension functions, MRA tight frame wavelets and connectivity properties, Adv. in Comp. Math., 18 (2003), 297-327.

[35] G. Pisier, Introduction to Operator Space Theory, London Math. Soc. Lecture Notes. Cambridge Univ. Press (2003).

[36] D. Speegle, Ph. D. thesis, Teaxs A\& M University, 1997.

[37] D. Speegle, The s -elementary wavelets are path-connected, Proc. Amer. Math. Soc., Vol 127 (1999), 223-233. 
[38] V. S. Varadarajan, Geometry of Quantum Theory, Second Edition, Springer-Verlag, New YorkBerlin, 1985.

[39] Wutam Consortium, Basic Properties of Wavelets, Journal of Fourier Analysis and Applica- tions, Vol 4, no. 4-5 (1998), 575-594

Department of Mathematics, University of Central Florida, Orlando, FL 32816

Department of Mathematics, Texas A\& M University, College Station, TX

E-mail address: dhan@pegasus.cc.ucf.edu

E-mail address: larson@math.tamu.edu 